\title{
NCTUns 5.0: A Network Simulator for IEEE 802.11(p) and 1609 Wireless Vehicular Network Researches
}

\author{
Shie-Yuan Wang \\ Department of Computer Science \\ National Chiao Tung University \\ Hsinchu, Taiwan \\ Email:shieyuan@csie.nctu.edu.tw
}

\author{
Chih-Che Lin \\ Department of Computer Science \\ National Chiao Tung University \\ Hsinchu, Taiwan \\ Email: linjc@cs.nctu.edu.tw
}

\begin{abstract}
NCTUns is a novel network simulator and emulator that has many unique features over traditional network simulators and emulators. It is an open-source software running on Linux and is being used by many researchers in the world. According to the NCTUns official web site (http://NSL.csie.nctu.edu.tw/nctuns.html), as of June 1, 2008, more than 11,546 people from 124 countries have registered and downloaded this software and these numbers are still fast increasing.

In its 5.0 release, NCTUns provides a complete implementation of the IEEE 802.11(p)/1609 standards defined for wireless vehicular networks. In this paper, we present the capabilities of NCTUns 5.0 focusing on its uses for IEEE 802.11(p)/1609 wireless vehicular network researches.
\end{abstract}

Index Terms-NCTUns, network simulation, IEEE 802.11p, IEEE 1609, vehicular network

\section{INTRODUCTION}

The NCTUns network simulator and emulator [1][2] is a valuable tool for network research, planning, and education. It is an open-source software running on Linux and provides an easy-to-use integrated GUI environment for users to efficiently conduct simulations/emulations. Many research institutes, industry companies, and universities are using it for various purposes. The authors of many journal papers, conference papers, Ph.D. dissertations, master theses, etc. have used it to conduct researches.

By adopting an innovative kernel re-entering simulation methodology [4], NCTUns provides many unique and important advantages over traditional network simulators and emulators. For example, NCTUns directly uses the real-life TCP/IP protocol stack in the Linux kernel to conduct simulations. As such, more realistic and high-fidelity simulation results are generated. In addition, any real-life application program can be run up on a simulated NCTUns device during simulation. This capability provides several unique and important advantages such as: 1) realistic network traffic can be generated by reallife applications on NCTUns to generate more realistic simulation results. 2) the performance of any real-life application can be easily evaluated on NCTUns under various simulated network conditions, and 3) any network application program developed for a NCTUns device can be directly run up on a real-life Linux device without any modification.

Recently, Intelligent Transportation Systems (ITS) research has gained much attention due to its abundant research and business potential. NCTUns has supported the simulation of ITS vehicular networks in its 4.0 release (released on July 25, 2007). In the 4.0 release, NCTUns supports 1) basic driver behavior models; 2) basic road network construction; and 3) simulation of road side unit (RSU) and on-board unit (OBU) devices that are equipped with a radio, which can be an IEEE 802.11(b) infrastructure mode radio, IEEE 802.11(b) ad-hoc mode radio, GPRS radio, or DVBRCST satellite radio. As compared with other ITS network simulators such as SUMO [5], NCTUns 4.0 provides users with a simulation environment in which traffic simulation and network simulation are tightly integrated. With this unique design, many advanced ITS researches in which a vehicle needs to quickly change its driving behavior after receiving a message from the network can be studied. Many unique advantages brought by this design have been presented in detail in [6].

In its 5.0 release (scheduled to be released in July, 2008), NCTUns greatly enhances its supports for ITS network simulations. Such new functions include 1) efficient node mobility control for very-large-scale vehicular networks; 2) automatic road network construction from the popular SHAPE-format map file; and, most importantly, 3) complete simulation of the IEEE 802.11(p)/1609 standards for wireless vehicular networks (WVN). The objective of this paper is to present the WVN capabilities of NCTUns 5.0.

\section{IMPLEMENTATION OF OBU AND RSU ON NCTUNS 5.0}

NCTUns 5.0 supports two types of nodes for IEEE 802.11(p)/1609 WVNs. One is "802.11(p) RSU" and the other is "802.11(p) OBU." The former is usually deployed at an intersection as a fixed relay node while the latter is usually installed on a vehicle as an end-user terminal device. Fig. 1 shows a snapshot of an IEEE 802.11(p)/1609 WVN created using NCTUns 5.0 GUI, where an 802.11(p) RSU is deployed 


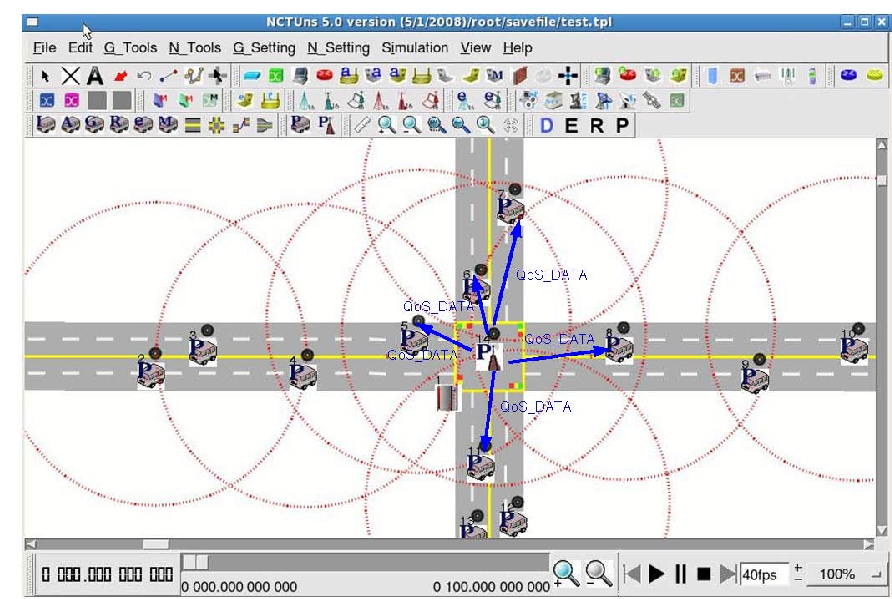

Fig. 1. Snapshot of an IEEE 802.11(p)/1609 WVN created using NCTUns 5.0

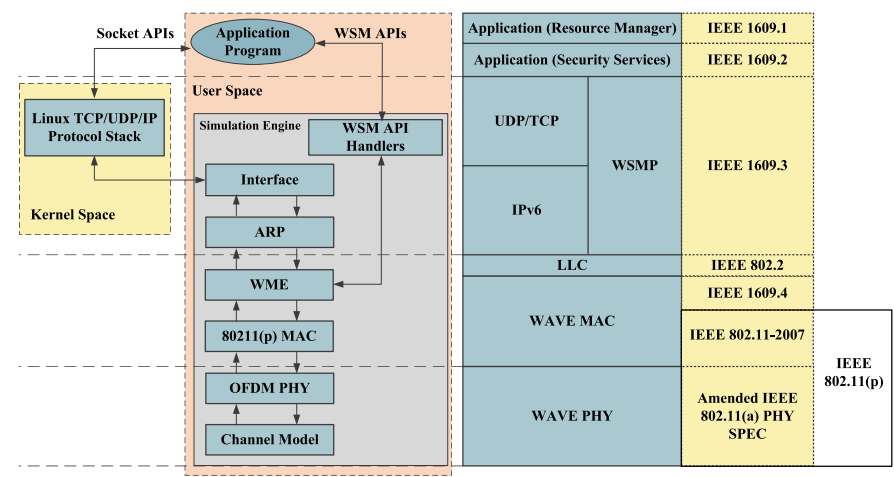

Fig. 2. Simulation architecture of NCTUns 5.0 for IEEE 802.11(p)/1609 WVN

at the intersection while several 802.11(p) OBUs are deployed on the simulated road network. Each dotted circle shown in the figure denotes the interference range of each RSU's or OBU's radio, while the colored arrows denote the 802.11(p) MAC-layer DATA frame that the RSU is transmitting.

These two types of nodes use the same protocol stack configuration, which is shown on the right of Fig. 2.

In the following, we present how NCTUns 5.0 realizes this IEEE $802.11(\mathrm{p}) / 1609$ protocol stack. The simulation architecture used by NCTUns 5.0 for this new network is shown on the left of Fig. 2, which is explained below.

\section{A. Application Program}

NCTUns directly executes a user-level application process to realize the functions of the application intended to be run on an IEEE 802.11(p)/1609 node. Such an application can transmit or receive 1) IP packets via the standard socket interface [6] or 2) WAVE short messages via the special WSMP Application Programming Interfaces (WSMP APIs) provided by the NCTUns simulation engine. The WSMP APIs are implemented using inter-process communication (IPC) between an application program and the simulation engine. NCTUns 5.0 does not implement the resource management and security functions defined by the IEEE 1609.1 and IEEE 1609.2 standards. This decision is made because these two standards are irrelevant to network performance evaluations.

\section{B. WSMP}

Unlike the TCP/UDP/IP protocol suite, which has been standardized for years and implemented by the Linux kernel, WSMP is a new network-layer protocol and has not been supported by Linux. As such, NCTUns 5.0 implements WSMP in its simulation engine as protocol modules. If in the future Linux implements WSMP as part of the standard socket system call library, any user-level application program can directly call socket system calls on NCTUns to transmit/receive WAVE Short Messages.

\section{MAC-layer and Physical-layer Modules}

The MAC-layer and physical-layer functions of the IEEE 802.11(p)/1609 network are implemented by simulation protocol modules. The WME functionality defined by the IEEE 1609.3 is implemented by the WME module, while the WAVEmode MAC-layer functions, such as channel switching and 802.11(e)-based prioritized channel accesses, are implemented by the "802.11(p) MAC" module. Finally, the characteristics of OFDM channels are simulated by the OFDM module.

\section{CONCLUSION}

NCTUns is a novel network simulator and emulator that directly uses real-life applications and Linux protocol stack to generate high-fidelity simulation results. In its 4.0 release, many features relevant to advanced ITS researches have been presented in [6]. In its 5.0 release, NCTUns provides a complete implementation of the emergent IEEE 802.11(p) and 1609 standards for wireless vehicular networks. With continuous improvements, NCTUns has become a very valuable tool for researching advanced ITS wireless vehicular networks.

\section{REFERENCES}

[1] S.Y. Wang et al., "The Design and Implementation of the NCTUns Network Simulator," Computer Networks, Vol. 42, Issue 2, June 2003, pp. 175-197.

[2] S.Y. Wang, C.L. Chou, C.C. Lin, "The Design and Implementation of the NCTUns Network Simulation Engine," Elsevier Simulation Modelling Practice and Theory, 15 (2007), p.p. 5781.

[3] The statistics report announced by the NCTUns official website, "http://nsl.cs.nctu.edu.tw/nctuns.htm," March 13, 2008.

[4] S.Y. Wang and H.T. Kung, "A New Methodology for Easily Constructing Extensible and High-Fidelity TCP/IP Network Simulators," Computer Networks, Vol. 40, Issue 2, October 2002, pp. 257-278.

[5] The SUMO traffic simulation package, available at http://sumo.sourceforge.net/index.shtml.

[6] S.Y. Wang et al., "NCTUns 4.0: An Integrated Simulation Platform for Vehicular Traffic, Communication, and Network Researches," 1st IEEE WiVec 2007 (International Symposium on Wireless Vehicular Communications, colocated with VTC 2007 Fall), Sept. 30 Oct. 1, 2007, Baltimore, MD, USA. 